\title{
Redox-active ferrocene-modified Cowpea mosaic virus nanoparticles $\uparrow$
}

\author{
Alaa A. A. Aljabali, ${ }^{a}$ J. Elaine Barclay, ${ }^{a}$ Julea N. Butt, ${ }^{b}$ George P. Lomonossoff ${ }^{a}$ and David J. Evans ${ }^{* a}$ \\ Received 18th May 2010, Accepted 11th June 2010 \\ First published as an Advance Article on the web 9th July 2010 \\ DOI: 10.1039/c0dt00495b
}

A naturally occurring nanoparticle, the plant virus Cowpea mosaic virus, can be decorated with ferrocene derivatives, of various linker lengths with amine and carboxylate groups, on the external surface using a range of conjugation strategies. The multiple, organometallic, redox-active ferrocene moieties on the outer surface of the virus are electrochemically independent with reduction potentials that span a potential window of $0.16 \mathrm{~V}$ that are dependent on the site of modification and the nature of the ferrocene derivative. The number of ferrocenes coupled to each virus ranges from about 100 to 240 depending upon the conjugation site and the linker length and these redox active units can provide multielectron reservoirs.

\section{Introduction}

Ferrocenes are known for their stability in aqueous aerobic media, their high thermal and photochemical stability, access to a large variety of derivatives, and their favourable electrochemical properties. Ferrocene-containing materials have been developed for applications in, for example, non-linear optics, ${ }^{1}$ molecular sensors and receptors, ${ }^{2-4}$ as ferromagnets ${ }^{5}$ and for electrochemistry and catalysis. ${ }^{4,6}$ Further, ferrocenes have been incorporated into dendrimers $^{7}$ and coupled to silica nanoparticles ${ }^{8}$ for a range of applications. We have previously communicated ${ }^{9}$ that the plant virus Cowpea mosaic virus (CPMV), a naturally occurring nanoparticle, can be decorated, by covalent attachment, with 240 redox-active ferrocenecarboxylate moieties. Electrochemical studies showed that the multiple redox centres behaved as electronically isolated units and that the electrochemical reaction was diffusion controlled. This was expected as the spatial separation, approximately 20-40 $\AA$, of the redox centres on the insulating CPMV platform was too long for electrochemical communication. If used as surface coatings of electrodes, CPMV-ferrocene derivatives are strong candidates for multielectron transfer mediators. As part of our continuing studies to develop CPMV-based sensors, we here describe how various coupling strategies can be employed to give a range of CPMV-ferrocene derivatives (Fig. 1) in which the separation distance between ferrocene and virus surface can be controlled. The ability to vary this distance will be of importance in the design of CPMV-based bioelectronic components and devices.

The virions of CPMV exhibit features ideal for exploitation in nanomaterial fabrication. Plant viruses present no biological hazard and are non-infectious towards other organisms. The particles, $28 \mathrm{~nm}$ in diameter, have discrete size and geometry with a high degree of symmetry and polyvalency and are extremely

${ }^{a}$ Department of Biological Chemistry, John Innes Centre, Norwich Research Park, Colney, Norwich, NR4 7UH, UK. E-mail: dave.evans@bbsrc.ac.uk; Fax: +44 (0) 1603450018

${ }^{b}$ School of Chemistry, University of East Anglia, Norwich, NR4 7TJ, UK $\dagger$ Electronic supplementary information (ESI) available: Dynamic light scattering measurements; plots of current versus the square route of the scan rate for the oxidation and reduction processes; data and calculation of number of bound ferrocenes by the Randles-Sevcik equation. See DOI: $10.1039 / \mathrm{c} 0 \mathrm{dt} 00495 \mathrm{~b}$ robust and well characterized. ${ }^{10,11}$ For example, they maintain their integrity at $60{ }^{\circ} \mathrm{C}(\mathrm{pH}$ 7) for at least one hour and at $\mathrm{pH}$ values from 3.5 to 10.0 at room temperature, and the virus can tolerate organic solvents such as dimethylsulfoxide (DMSO) or dimethylformamide (DMF) at concentrations up to $50 \%$ for at least several hours. ${ }^{12} \mathrm{CPMV}$ virions are formed by 60 copies of the asymmetric unit, which is comprised of two different types of coat protein, the small $(\mathrm{S}, 24 \mathrm{kDa})$ and the large $(\mathrm{L}, 42 \mathrm{kDa})$ subunit. Due to its topology the virion can be regarded as being like a large dendrimer, the multiple copies of the asymmetric unit provide regularly spaced attachment units, facilitating the coupling and presentation of different moieties on the exterior surface. It has previously been shown that both wild-type CPMV and CPMV mutants can be functionalised and decorated with a variety of molecules ${ }^{13}$ demonstrating the feasibility of utilising CPMV as a molecular assembly in bionanotechnology.

\section{Experimental}

\section{General instrumentation}

UV-visible spectra were recorded using a Perkin Elmer Lambda $25 \mathrm{UV}$-visible spectrometer and UVWINLab software; ${ }^{1} \mathrm{H}$-NMR spectra on a Bruker Avance III $400 \mathrm{MHz}$; mass spectra using a DecaXPplus ion trap; transmission electron microscopy (TEM) images on a FEI Tecnai 20 TEM, FEI UK Ltd, Cambridge and dynamic light scattering (DLS) was measured on a DynaPro Titan, Wyatt Technology Corporation. Native agarose gel electrophoresis was performed on $1.2 \%$ agarose gels; CPMV particles $(10 \mu \mathrm{g})$ were loaded (added loading dye, MBI Fermentas, Inc.) and after migration in the electric field, bands were detected by intercalation of ethidium bromide and visualisation under UV light.

\section{Cowpea mosaic virus (CPMV)}

The propagation and purification of wild-type CPMV virions were performed by standard procedures. ${ }^{14}$ Purified virions were stored at $4{ }^{\circ} \mathrm{C}$ in $10 \mathrm{mM}$ sodium phosphate buffer $\mathrm{pH}$ 7.0. The concentration of purified virions was determined photometrically; the molar extinction coefficient of CPMV at a wavelength of $\lambda=$ $260 \mathrm{~nm}$ is $\varepsilon=8.1 \mathrm{~mL} \mathrm{mg}^{-1} \mathrm{~cm}^{-1}$. 

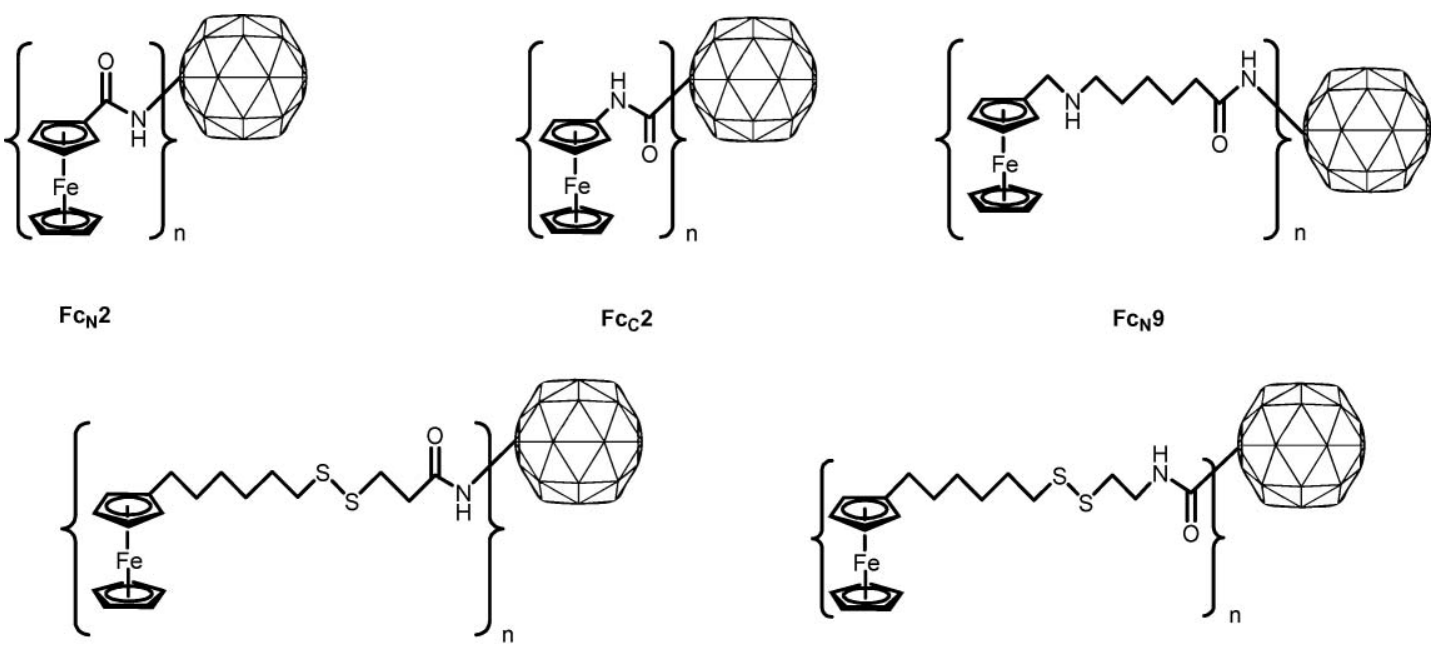

$c_{c} 2$

$\mathrm{Fc}_{\mathrm{N}} 9$

$\mathrm{FC}_{\mathrm{N}} 12$

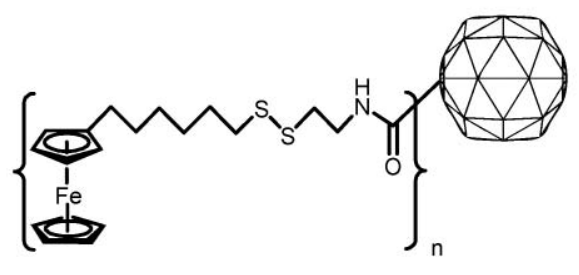

$\mathrm{Fc}_{\mathrm{C}}{ }^{12}$

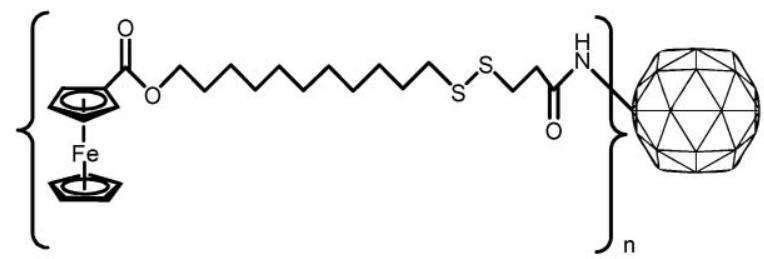

$\mathrm{FC}_{\mathrm{N}} 19$

Fig. 1 Ferrocene-CPMV derivative nomenclature. Subscript: N represents linkage to virus surface amine, $\mathrm{C}$ represents linkage to surface carboxylate; numeral gives number of atoms in link between ferrocene and virus surface, where virus surface is taken to be the $\varepsilon \mathrm{C}$ of the lysine side chain, the $\beta \mathrm{C}$ of aspartate and the $\chi \mathrm{C}$ of glutamate.

\section{Reagents and ferrocene derivatives}

All general reagents and 6-(ferrocenyl)hexanethiol were purchased from Sigma-Aldrich UK and used without further purification. 1-Ethyl-3-(3-dimethlyaminopropyl)carbodiimide hydrochloride (EDC) was purchased from Novabiochem, $N$-hydroxysuccinimide (NHS) from Fluka, $N$-succinimidyl-3-(2-pyridyldithio)propionate (SPDP) from Pierce, 2-(2-pyridinyldithio)ethaneamine hydrochloride (PDEA) from GE Healthcare and aminoferrocene from TCI Europe. $N$-(Ferrocenylmethyl)-6-aminocaproicacid ${ }^{15}$ and 11-(ferrocenylcarbonyloxy)undecanethiol ${ }^{16}$ were synthesised by published methods and their purity confirmed by ${ }^{1} \mathrm{H}-\mathrm{NMR}$ and mass spectrometry.

\section{Coupling of ferrocene derivatives to CPMV}

EDC/NHS method. $N$-(Ferrocenylmethyl)-6-aminocaproic acid, FcAC, and aminoferrocene, $\mathrm{FcNH}_{2}$, (Fig. 2) were coupled to CPMV surface amine groups and CPMV surface carboxylate groups, respectively, by our standard EDC/NHS method. ${ }^{9,17}$ After stirring gently overnight at $4{ }^{\circ} \mathrm{C}$ the $\mathrm{CPMV}$-ferrocene conjugates were purified either by gel filtration on a Sephacryl S-500 column (GE Healthcare) or by dialysis using $100 \mathrm{kDa}$ molecular weight cut-off membranes (Float-A-Lyzer G2, Spectrum Laboratories) against $10 \mathrm{mM}$ sodium phosphate buffer. The samples were concentrated on $100 \mathrm{kDa}$ cut-off columns (Millipore) and the concentration of ferrocene-virus conjugate was determined

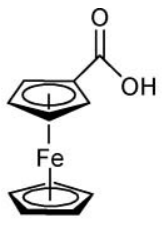

(a)

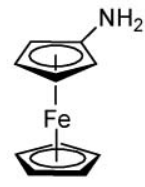

(b)

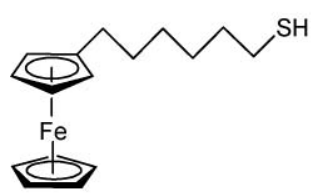

(c)

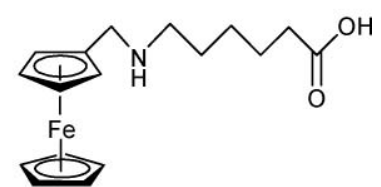

(d)

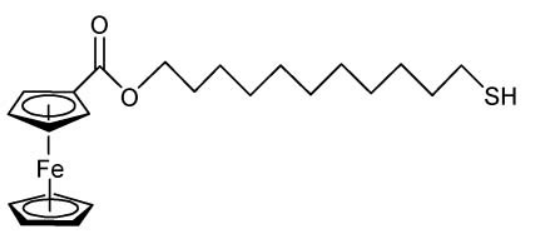

(e)

Fig. 2 Ferrocene reagents used in this study. (a) Ferrocene carboxylic acid, $\mathrm{FcCOOH}$; (b) aminoferrocene, $\mathrm{FcNH}_{2}$; (c) 6-(ferrocenyl)hexanethiol, Fc6SH; (d) $N$-(ferrocenylmethyl)-6-aminocaproic acid, FcAC; (e) 11-(ferrocenylcarbonyloxy)undecanethiol, Fc11SH. 
photometrically at $260 \mathrm{~nm}$. Agarose gel electrophoresis, TEM and DLS confirmed the integrity of the modified virus.

SPDP method. The ferrocene-thiols 6-(ferrocenyl)hexanethiol, Fc6SH, and 11-(ferrocenylcarbonyloxy)undecanethiol, Fc11SH, (Fig. 2) were coupled to CPMV surface amine groups using SPDP. CPMV particles (10-20 $\mathrm{mg} \mathrm{mL}^{-1}$ ), suspended in $10 \mathrm{mM}$ sodium phosphate buffer $\mathrm{pH}$ 7.0, were incubated with a solution of SPDP (1000 molar excess) in DMSO, the final concentration of DMSO was adjusted to $20 \%(\mathrm{v} / \mathrm{v})$. The reaction was stirred gently overnight at $4{ }^{\circ} \mathrm{C}$. The modified CPMV particles were dialyzed using $100 \mathrm{kDa}$ molecular weight cut-off membranes against $10 \mathrm{mM}$ sodium phosphate buffer and then concentrated using $100 \mathrm{kDa}$ cut-off columns. 3-(2-Pyridyldithio)propionateCPMV modified particles were incubated overnight at $4{ }^{\circ} \mathrm{C}$ with ferrocene-thiol (1000 molar excess) in either DMSO or DMF; the final concentration of organic solvent was adjusted to $20 \%(\mathrm{v} / \mathrm{v})$. Ferrocene-CPMV particles were purified by gel filtration (Sephacryl S-500 column) and the eluted fractions were collected and concentrated on $100 \mathrm{kDa}$ cut-off columns. The ferrocene-CPMV particle integrity was established by agarose gel electrophoresis, TEM and DLS.

PDEA method (ref. 18). The ferrocene-thiol Fc6SH (Fig. 2) was coupled to CPMV surface carboxylate groups with PDEA. To pre-activate the surface carboxylate groups, CPMV particles (10 $\mathrm{mg} \mathrm{mL}^{-1}$ ), suspended in $10 \mathrm{mM}$ sodium phosphate buffer $\mathrm{pH} 7.0$, were reacted with a freshly prepared solution of EDC (1000 molar excess) and NHS (4000 molar excess) for $2 \mathrm{~h}$ at ambient temperature with gentle stirring. The pre-activated particles were purified on PD-10 Desalting Columns (GE Healthcare). The eluted sample was reacted with a solution of PDEA (1000 molar excess) in Milli-Q water overnight, purified on a $100 \mathrm{kDa}$ cut-off column and washed thoroughly to remove any unreacted materials. PDEA-derivatised CPMV particles were reacted overnight at $4{ }^{\circ} \mathrm{C}$ with Fc6SH (1000 molar excess) dissolved in DMSO, the final DMSO concentration being adjusted to $20 \%(\mathrm{v} / \mathrm{v})$, purified by gel filtration (Sephacryl S-500 column), and the eluted fractions collected and concentrated on $100 \mathrm{kDa}$ cut-off columns. The ferrocene-CPMV particle integrity was established by agarose gel electrophoresis, TEM and DLS.

\section{Electrochemical measurements}

Cyclic voltammetry was conducted with an AUTOLAB PGSAT 30 potentiostat in a conventional three-electrode cell with the sample in $10 \mathrm{mM}$ sodium phosphate buffer $\mathrm{pH} 7.0$ at room temperature $\left(c a .25^{\circ} \mathrm{C}\right)$. The working electrode was a pyrolytic graphite edge (PGE) disc with a diameter of $3 \mathrm{~mm} ; \mathrm{Ag} / \mathrm{AgCl}$ (saturated $\mathrm{KCl}$ ) was the reference electrode and platinum wire the counter electrode. Analysis of the free ferrocene moieties was performed with a concentration of 5-10 $\mathrm{mM}$ (with $10 \%$ DMSO or DMF as necessary for solubility). Modified virions were typically studied at concentrations of 1 to $11 \mathrm{mg} \mathrm{mL}^{-1}$. For each sample cyclic voltammograms were recorded at scan rates from 5 to $200 \mathrm{mV} \mathrm{s}^{-1}$ in duplicate sets with the working electrode polished by abrasion with an aqueous slurry of $0.3 \mu \mathrm{m} \mathrm{Al}_{2} \mathrm{O}_{3}$ immediately prior to measuring each set. Consecutive voltammograms at each scan rate overlaid each other from the fourth scan onwards and these 'steady-state' voltammograms were analysed to determine peak potentials $\left(E_{\mathrm{p}}{ }^{\mathrm{a}}\right.$ and $E_{\mathrm{p}}{ }^{\mathrm{c}}$ for the anodic and cathodic scans respectively) and peak current magnitudes $\left(i_{\mathrm{p}}{ }^{\mathrm{a}}\right.$ and $i_{\mathrm{p}}{ }^{\mathrm{c}}$ for the anodic and cathodic scans, respectively) at each scan rate. Analysis of $20 \mathrm{mV} \mathrm{s}^{-1}$ voltammetry performed at various times during the collection of each set of scan rates confirmed that the response was independent of time. Plots of peak current versus the square root of scan rate were found to be linear. The gradients were determined by linear regression and analysed by the Randles-Sevcik equation to determine the concentration of ferrocene in the sample and ultimately the number of ferrocene moieties per virus particle., ${ }^{9,17,19}$

\section{Results and discussion}

CPMV surface amine groups, arising from exposed lysines, can be readily coupled to ferrocene-carboxylate derivatives using EDC/NHS methods ${ }^{9}$ and to ferrocene-thiols using SPDP reagent. The SPDP reagent was first coupled to the amine groups of the virus by reaction with the succinimidyl group of the reagent. The 3-(2-pyridyldithio)propionate-CPMV derivative that was generated was isolated and purified. The pyridyl disulfide group of this derivative was then reacted with ferrocene-thiol; pyridine was liberated and a disulfide bond was formed between the ferrocene and the modified virus (Fig. 1). Further, CPMV surface carboxylate groups, from exposed aspartic and glutamic acids, can be coupled to aminoferrocene, using EDC/NHS, or to ferrocenethiol, Fc6SH, with PDEA (Fig. 1). Prior to coupling of PDEA to the virus, the surface carboxylates are pre-activated with NHS, these react with the amine group on the PDEA reagent to form a stable amide bond. Ferrocene-thiol can then be coupled to the modified CPMV by thiol-disulfide exchange. In all cases the ferrocene reagents are used in a large excess to ensure maximum coupling. Virus particles were recovered in approximately $70-80 \%$ yield based on initial wild-type concentration. In each case, and as described below, successful ferrocenylation of the virus capsid with retention of virus integrity was established by uranyl acetate negatively stained TEM, DLS, native gel electrophoresis and cyclic voltammetry.

The TEM shows that the virus particles remain intact and that they are monodisperse (for example, Fig. 3). DLS measurements gave the average diameter of the ferrocene-modified virus particles and confirm monodispersity (Table 1). The increase in spherically averaged outer diameter compared to native CPMV particles $(28.4 \mathrm{~nm})$ is consistent with decoration with ferrocene. The measured polydispersity indicates similar decoration of each individual particle as shown by the narrow size distribution. Preparations of CPMV particles resolve into several bands during agarose gel electrophoresis as a consequence of their differing RNA contents and surface charge (Fig. 4). Ferrocene-CPMV derivatives with shorter

Table 1 Diameter and polydispersity of ferrocene-CPMV as determined by dynamic light scattering

\begin{tabular}{lll}
\hline Ferrocene-CPMV & Diameter $(\mathrm{nm})$ & Polydispersity $(\%)$ \\
\hline $\mathrm{Fc}_{\mathrm{N}} 2$ & 29.8 & 24.5 \\
$\mathrm{Fc}_{\mathrm{N}} 9$ & 30.0 & 10.7 \\
$\mathrm{Fc}_{\mathrm{N}} 12$ & 32.0 & 20.6 \\
$\mathrm{Fc}_{\mathrm{N}} 19$ & 33.0 & 20.8 \\
$\mathrm{Fc}_{\mathrm{C}} 2$ & & \\
$\mathrm{Fc}_{\mathrm{C}} 12$ & 29.4 & 11.8 \\
\hline
\end{tabular}


Table 2 Reduction potential $\left(E_{1 / 2}\right)$ of ferrocene and ferrocene-CPMV $v s . \mathrm{Ag} / \mathrm{AgCl}$ (saturated $\mathrm{KCl}$ ) and the average number of ferrocenes per $\mathrm{CPMV}$ virion as calculated from the linear plots of current versus the square-root of the scan rate and the Randles-Sevcik equation

\begin{tabular}{lllll}
\hline Ferrocene reagent & $E_{1 / 2}(\mathrm{~V})( \pm 0.015)$ & Ferrocene-CPMV & $E_{1 / 2}(\mathrm{~V})( \pm 0.015)$ & Number of ferrocenes per virion $( \pm 5 \%)$ \\
\hline FcCOOH & 0.322 & $\mathrm{Fc}_{\mathrm{N}} 2$ & 0.233 & $240^{9}$ \\
$\mathrm{FcAC}$ & 0.425 & $\mathrm{Fc}_{\mathrm{N}} 9$ & 0.373 & 246 \\
$\mathrm{Fc6SH}$ & 0.455 & $\mathrm{Fc}_{\mathrm{N}} 12$ & 0.325 & 250 \\
$\mathrm{Fc11SH}$ & 0.450 & $\mathrm{Fc}_{\mathrm{N}} 19$ & 0.344 & 193 \\
& & & 0.210 & 174 \\
$\mathrm{FcNH}$ & & $\mathrm{Fc}_{\mathrm{C}} 2$ & 0.320 & 101 \\
$\mathrm{Fc6SH}$ & 0.380 & $\mathrm{Fc}_{\mathrm{C}} 12$ & & \\
\hline
\end{tabular}

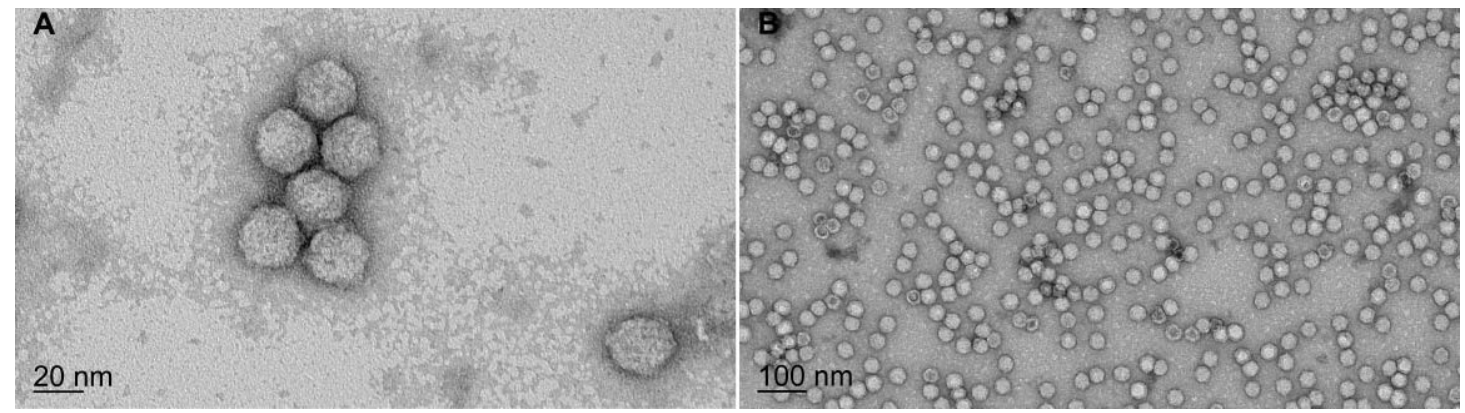

Fig. 3 Transmission electron microscopy images of particles on carbon-coated copper grids after staining with uranyl acetate. (A) Wild-type CPMV; (B) Ferrocene-CPMV, $\mathrm{Fc}_{\mathrm{C}} 12$.
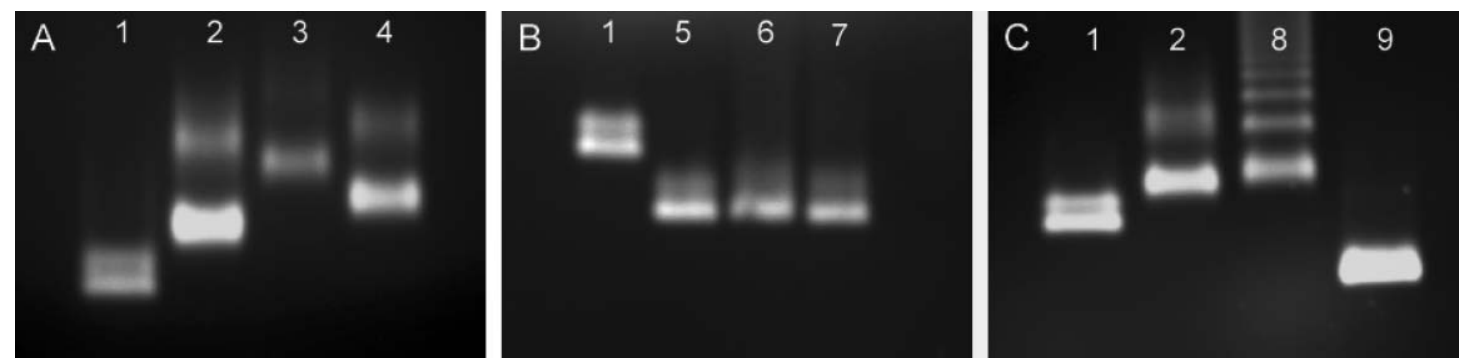

Fig. 4 Wild-type and ferrocene-CPMV particles on a 1.2\% agarose gel. (A) Lane 1, wild-type CPMV; 2, CPMV-NHS ester; 3, Fc 2 ; 4, Fc $\mathrm{C}_{\mathrm{N}}$ 9. (B) Lane 1, wild-type CPMV; 5, 3-(2-pyridyldithio)propionate-CPMV derivative; 6, $\mathrm{Fc}_{\mathrm{N}} 12 ; 7, \mathrm{Fc}_{\mathrm{N}}$ 19. (C) Lane 1, wild-type CPMV; 2, CPMV-NHS ester; 8, 2-(2-pyridinyldithio)ethaneamide-CPMV derivative; 9, $\mathrm{Fc}_{\mathrm{C}} 12$.

linker length, whether coupled through amine or carboxylate groups, $\mathrm{Fc}_{\mathrm{C}} 2$ and $\mathrm{Fc}_{\mathrm{N}}$, exhibit retarded mobility of the ferrocenemodified virus particles compared to wild-type CPMV (Fig. 4), as has been previously observed for $\mathrm{Fc}_{\mathrm{N}} 2,{ }^{9}$ and is consistent with an increase in size and, therefore, decoration of the virions with ferrocene molecules. Surprisingly, ferrocene-CPMV particles with longer linker length and coupled via a disulfide linkage, $\mathrm{Fc}_{\mathrm{N}} 12$, $\mathrm{Fc}_{\mathrm{C}} 12$ and $\mathrm{Fc}_{\mathrm{N}} 19$, show enhanced mobility toward the anode as does a 3-(2-pyridyldithio)propionate-CPMV derivative positive control (Fig. 4). This unexpected behaviour is thought to arise as a consequence of a combination of size and charge effects perturbing mobility through the gel. However, all migrate to a different extent than wild-type confirming that the particles have been modified.

Cyclic voltammetry established that the modified-CPMV are redox-active nanoparticles, (e.g. Fig. 5 and 6). The ratio of the peak currents, $i_{\mathrm{p}}{ }^{\mathrm{a}} / i_{\mathrm{p}}{ }^{\mathrm{c}}$, is close to unity showing the ferrocene/ferrocenium couple is electrochemically reversible. The peak currents were proportional to the square root of the scan rate, $v^{1 / 2}$, showing that oxidation and reduction were diffusion controlled (e.g. Fig. 5, Fig. 6 and ESI $\dagger$ ). This allowed the concentration of ferrocene molecules in the ferrocene conjugated CPMV samples to be determined using the Randles-Sevcik equation. ${ }^{9,17,19}$ Comparing the concentration of ferrocene to that of the CPMV itself determined photometrically, the number of ferrocene molecules attached to CPMV were calculated as summarised in Table 2.

The asymmetric unit of CPMV can be maximally labelled at four of the five lysines presented on the outer surface (Lys 38, 82 of S subunit and Lys 34, 99, 199 of L subunit). ${ }^{20}$ This provides a maximum of 240 addressable lysine sites on the CPMV virion surface., ${ }^{9,132,21} \mathrm{Fc}_{\mathrm{N}} 2, \mathrm{Fc}_{\mathrm{N}} 9$ and $\mathrm{Fc}_{\mathrm{N}} 12$ show maximum coverage. However, when the linker length is increased further, as in $\mathrm{Fc}_{\mathrm{N}} 19$, there is a decrease in the number of ferrocenes bound from about 240 to 193. Similarly, the maximum number of addressable carboxylates at the virion surface has been determined to be 180 of the 420 aspartate and glutamate residues on the outer surface (Asp 26, 44, 45, 85, and Glu 135 on the S subunit, and Asp 273 and Glu 319 of the L subunit). ${ }^{17}$ The ferrocene-CPMV with a short link, $\mathrm{Fc}_{\mathrm{C}} 2$, shows maximum coverage, whereas that with a 

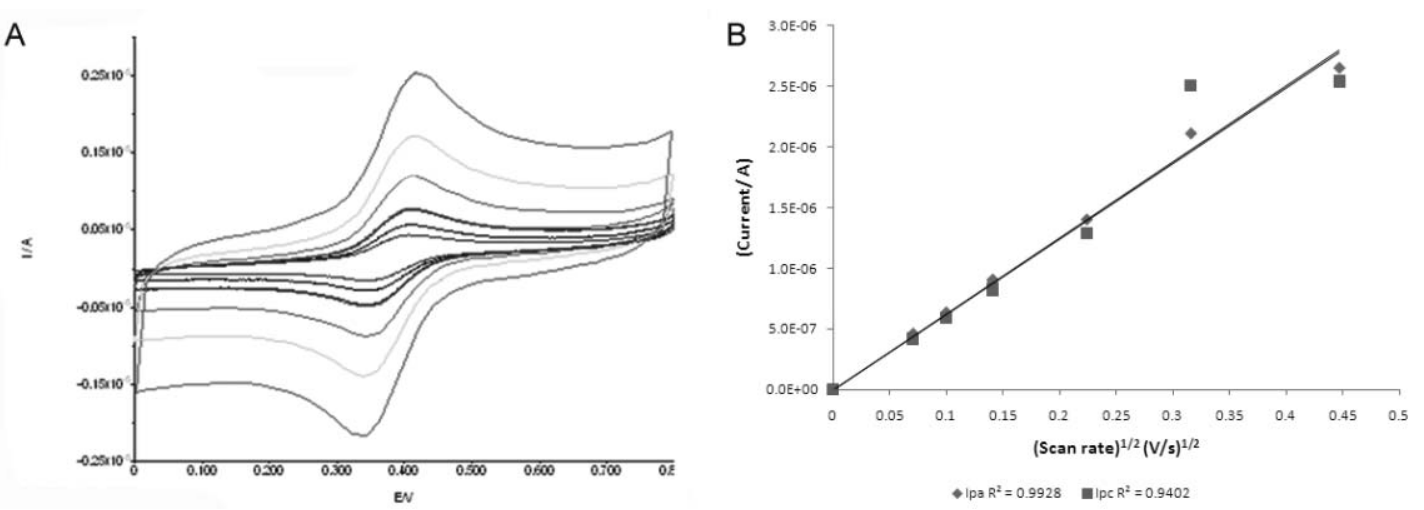

Fig. 5 (A) Cyclic voltammograms of $\mathrm{Fc}_{\mathrm{N}} 9$ at scan rates from $5 \mathrm{mV}$ to $200 \mathrm{mV} \mathrm{s}^{-1}$; (B) variation of peak current with the square root of the scan rate.
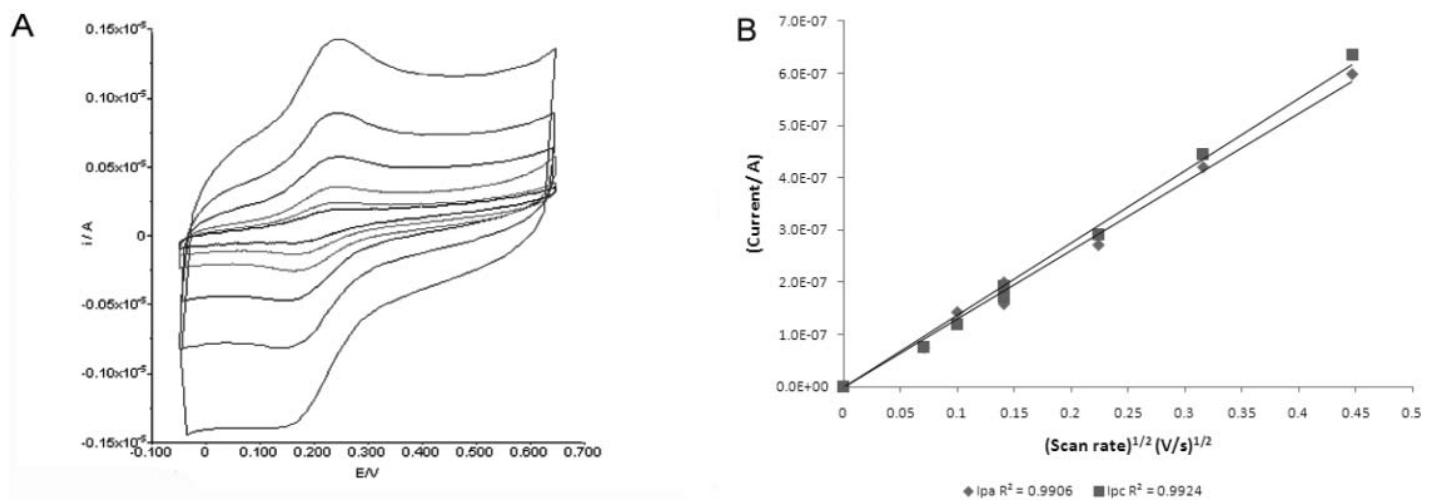

Fig. 6 (A) Cyclic voltammograms of $\mathrm{Fc}_{\mathrm{C}} 2$ at scan rates from $5 \mathrm{mV}$ to $200 \mathrm{mV} \mathrm{s}^{-1}$; (B) variation of peak current with the square root of the scan rate.

longer linker, $\mathrm{Fc}_{\mathrm{C}} 12$, exhibits reduced coverage. The decrease in the number of bound ferrocenes as a function of linker length is likely to be a consequence of the longer linker length molecules folding back onto the surface and blocking some of the other coordination sites. It is less likely to be a consequence of the different coupling strategies employed, as the conditions used would favour maximum coverage. For example, the most reactive lysine is Lys 38 and its closest neighbour is Lys $99,{ }^{21}$ the second most reactive at a distance of $c a$. $18 \AA$. In $\mathrm{Fc}_{\mathrm{N}} 19$ the distance between the surface of the virion and the ferrocene is approximately $26 \AA$, compared with, for example, $19.5 \AA$ in $\mathrm{Fc}_{\mathrm{N}} 9$; consequently, in $\mathrm{Fc}_{\mathrm{N}} 19$, reaction at Lys99 is sterically hindered so there are, within error, sixty less ferrocenes coupled to the virion surface.

Reduction potentials $\left(E_{1 / 2}\right)$ determined for the free and conjugated ferrocene moieties from the average peak potentials are shown in Table 2. Conjugation to the virus results in destabilisation of the ferrocenium form as reflected in the lower $E_{1 / 2}$ for the conjugated form. In each case, and for all scan rates studied, the peak separation $\left|E_{\mathrm{p}}{ }^{\mathrm{a}}-E_{\mathrm{p}}{ }^{\mathrm{c}}\right|$ ranged from 55 to $70( \pm 5)$ $\mathrm{mV}$ which is close to the theoretical value expected for a oneelectron process $\left(59 \mathrm{mV}\right.$ at $\left.25^{\circ} \mathrm{C}\right)$. Thus, the voltammetry indicates that the ferrocenyl centres coupled to the CPMV surface behave as independent and electronically isolated units with apparently homogeneous redox properties. Electronic isolation is likely to reflect a spacing of the ferrocene units across the virion surface that prevents their close approach for electron transfer. Given that the ferrocene units are conjugated to multiple environments on the surface of the asymmetric unit the homogeneity of the redox properties suggests the ferrocenes exhibit similar reduction potentials in all of these sites.

\section{Conclusion}

We have shown that both amine and carboxylate groups on the surface of CPMV can be decorated with ferrocene, with various linker lengths, utilising a range of conjugation strategies. The number of ferrocenes, determined electrochemically, per virus particle decreases with the length of the linker. The ferrocene moieties on the outer surface of the virus are electrochemically independent and can act as multielectron reservoirs. The modified virus particles provide a selection of redox active nanocomponents. We are currently exploring how these can be assembled onto solid electrode surfaces where they may act as electron transfer mediators for the development of redox catalysts, biosensors and nanoelectronic devices.

\section{Acknowledgements}

This work was supported by the Biotechnology and Biological Sciences Research Council, UK [Core Strategic Grant to the John Innes Centre, G.P.L. and D.J.E. and JIC DTG, A. A. A. A.]. Kim Findlay and Sue Bunnewell are thanked for technical assistance. 


\section{References}

1 (a) A. Togni and G. Rihs, Organometallics, 1993, 12, 3368-3372; (b) F. Yang, X.-L. Xu, Y.-H. Gong, W.-W. Qiu, Z.-R. Sun, J.-W. Zhou, P. Audebert and J. Tang, Tetrahedron, 2007, 63, 9188-9194.

2 (a) G. R. Newkome, E. F. He and C. N. Moorefield, Chem. Rev., 1999, 99, 1689-1746; (b) E. C. Constable, Angew. Chem., Int. Ed. Engl., 1991, 30, 407-408.

3 (a) J. L. Lopez, A. Tarraga, A. Espinosa, M. D. Velasco, P. Molina, V. Lloveras, J. Vidal-Gancedo, C. Rovira, J. Veciana, D. J. Evans and K. Wurst, Chem.-Eur. J., 2004, 10, 1815-1826; (b) P. Molina, A. Tarraga and A. Caballero, Eur. J. Inorg. Chem., 2008, 34013417.

4 Ferrocenes. Ligands, Materials and Biomolecules, ed. P. Stepnicka, John Wiley \& Sons Ltd, Chichester, 2008.

5 (a) M. L. H. Green, S. R. Marder, M. E. Thompson, J. A. Bandy, D. Ploor, P. V. Kolinsky and R. J. Jones, Nature, 1987, 330, 360362; (b) H. Yang, W. Jiang and Y. Lu, Mater. Lett., 2007, 61, 1439 1442.

6 (a) Electron Transfer in Chemistry, ed. V. Balzani, Wiley-VCH Verlag GmbH, Weinheim, 2001; (b) Ferrocenes-Homogeneous CatalysisOrganic Synthesis-Materials Science, ed. A. Togni and T. Hayashi, VCH, Weinheim, 1995.

7 D. Astruc, C. Ornelas and J. Ruiz, Chem.-Eur. J., 2009, 15, 8936-8944.

8 C. A. Beasley and R. W. Murray, Langmuir, 2009, 25, 10370-10375.

9 N. F. Steinmetz, G. P. Lomonossoff and D. J. Evans, Small, 2006, 2 , 530-533.
10 T. Lin, Z. Chen, R. Usha, C. V. Stauffacher, J. B. Dai, T. Schmidt and J. E. Johnson, Virology, 1999, 265, 20-34.

11 G. P. Lomonossoff and J. E. Johnson, Prog. Biophys. Mol. Biol., 1991, $\mathbf{5 5}, 107-137$.

12 Q. Wang, T. Lin, L. Tang, J. E. Johnson and M. G. Finn, Angew. Chem., Int. Ed., 2002, 41, 459-462.

13 (a) N. F. Steinmetz and D. J. Evans, Org. Biomol. Chem., 2007, 5, 28912902; (b) D. J. Evans, J. Mater. Chem., 2008, 18, 3746-3754; (c) Viruses and Nanotechnology. Current Topics in Microbiology and Immunology, vol. 327, ed. M. Manchester and N. F. Steinmetz, Springer-Verlag, Berlin, 2008; (d) D. J. Evans, Biochem. Soc. Trans., 2009, 37, 665-670.

14 J. Wellink, Methods Mol. Biol., 1998, 81, 205-209.

15 C. Padeste, A. Grubelnik and L. Tiefenauer, Biosens. Bioelectron., 2000, $15,431-438$.

16 C. E. D. Chidsey, C. R. Bertozzi, T. M. Putvinski and A. M. Mujsce, J. Am. Chem. Soc., 1990, 112, 4301-4306.

17 N. F. Steinmetz, G. P. Lomonossoff and D. J. Evans, Langmuir, 2006, 22, 3488-3490.

18 F. Frederix, K. Bonroy, G. Reekmans, W. Laureyn, A. Campitelli, M. A. Abramov, W. Dehaen and G. Maes, J. Biochem. Biophys. Methods, $2004, \mathbf{5 8}, 67-74$

19 Electrochemistry at Solid Electrodes, ed. R. N. Adams, Marcel Dekker, New York, 1969.

20 Q. Wang, E. Kaltgrad, T. Lin, J. E. Johnson and M. G. Finn, Chem. Biol., 2002, 9, 805-811.

21 A. Chatterji, W. F. Ochoa, M. Paine, B. R. Ratna, J. E. Johnson and T. Lin, Chem. Biol., 2004, 9, 855-863. 